\title{
Design and control of a heavy material handling manipulator for agricultural robots
}

\author{
Satoru Sakai • Michihisa Iida $\cdot$ Koichi Osuka • \\ Mikio Umeda
}

Received: 24 April 2007 / Accepted: 28 January 2008 / Published online: 14 February 2008

(C) The Author(s) 2008. This article is published with open access at Springerlink.com

\begin{abstract}
In this paper, we propose a manipulation system for agricultural robots that handle heavy materials. The structural systems of a mobile platform and a manipulator are selected and designed after proposing new knowledge about agricultural robots. Also, the control systems for these structural systems are designed in the presence of parametric perturbation and uncertainty while avoiding conservative results. The validity of both the structural and control systems is confirmed by conducting watermelon harvesting experiments in an open field. Furthermore, an explicit design procedure is confirmed for both the structural and control systems and three key design tools are clarified.
\end{abstract}

Keywords Agricultural robots · Manipulator · Robust control $\cdot$ Evaluation index

\section{Introduction}

In the field of agriculture, various operations for handling heavy materials must be performed. For example, in vegetable cropping, workers handle heavy vegetables during the harvest season. Additionally, in organic farming, which is rapidly gaining popularity, workers handle heavy compost

\footnotetext{
S. Sakai $(\bowtie)$

Department of Electronics and Mechanical Engineering,

Chiba University, Chiba, Japan

e-mail: satorusakai@ faculty.chiba-u.jp

M. Iida $\cdot$ M. Umeda

Graduate School of Agriculture, Kyoto University, Kyoto, Japan

K. Osuka

Department of Mechanical Engineering, Kobe University, Kobe, Japan
}

bags during the fertilizing season. These operations are dull, repetitive, and require strength and skill on the part of the workers.

A great deal of research on and development of agricultural robots took place in the 1980s. Kawamura et al. (1984) developed a fruit-harvesting robot for orchards. Grand et al. (1987) developed an apple-harvesting robot. Their studies were followed by others (e.g., Kondo and Ting 1998; Hwang and Kim 2003; Mario and Reina 2007; Tokunaga et al. 1999; Henten et al. 2003) including Edan's study (Edan et al. 2000) and our studies (Sakai et al. 2002, 2003, 2004, 2005, 2007). Many of these studies specifically examine the structural system design (e.g., machine or circuit design, camera configuration) of robots and report the realization of basic actions in actual open fields. Recently, Martinet and co-workers (Lenain et al. 2006; Fang et al. 2005) reasonably discussed the control system design of agricultural vehicles in sliding conditions. Taken together, these studies specifically address only one structural system or one control system of agricultural robots. Few papers have discussed explicit design procedures for both structural systems and control systems. However, many agricultural robots are currently not in the commercialization or diffusion stages. Instead, they remain in the research and development stages. It is thus important to support further research and development to improve the performance and reduce the initial cost of these robots.

Apart from some developing components such as advanced vision, it remains unclear how much the existing (and implicit) design procedures can be improved. This situation is serious because it is also unclear whether there are design tools that evaluate the possible improvements to the performance and initial cost. In order to clarify the status of these design tools, we need to confirm an explicit design procedure for both the structural and control systems. 


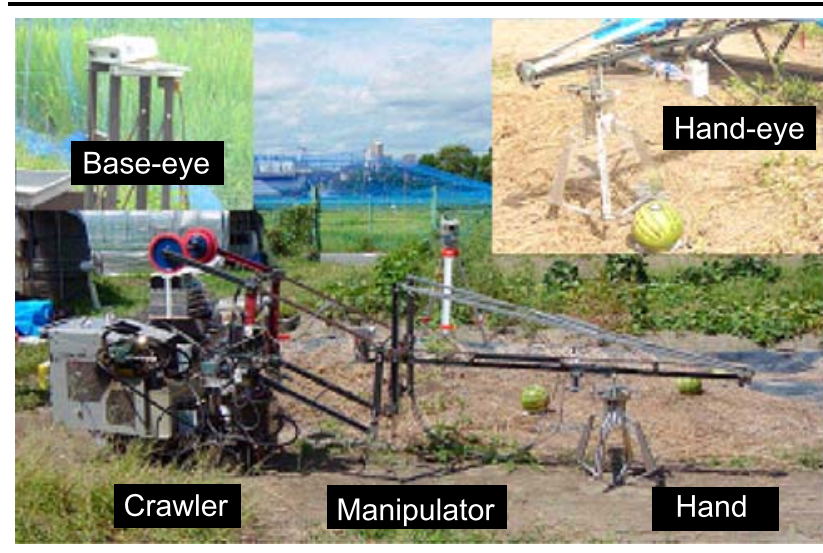

Fig. 1 A heavy material handling agricultural robot

In this paper, we report the realization of a heavy material handling manipulator for agricultural robots in Fig. 1. More precisely, the structural systems of a mobile platform and a manipulator with a hand are selected and designed after proposing new knowledge about agricultural robots. Also, the control systems are designed in the presence of parametric perturbation and uncertainty while avoiding conservative results. The validity of both systems is confirmed by performing field experiments in an open field. These experimental results are the most important contribution of this paper. Our field results demonstrate that the total operation time and success rate are comparable to those of skilled workers.

Furthermore, an explicit design procedure is confirmed to clarify the status of the design tools that evaluate the possible improvements of agricultural robots, which is another important contribution of this paper.

This paper is organized as follows. Section 2 proposes a new performance index of agricultural robots. Section 3 discusses the selection and design of the structural system for the mobile platform and the manipulator. In Sect. 4 the control system is designed in an analytical way. Section 5 confirms the validity of the systems by performing experiments in an outdoor field. Section 6 concludes this paper.

\section{Global performance index}

In this section, we discusses evaluation indexes for the global performance of agricultural robots. In general, we cannot start any reasonable design without using performance indexes. Many performance indexes have been proposed for general robots. For example, manipulability (Yoshikawa 1985) is a well-known index for structural systems. There are, however, some indexes that have been proposed specifically for agricultural robots. For example, space for obstacle avoidance (Kondo and Ting 1998) is used for collision avoidance between agricultural robots and

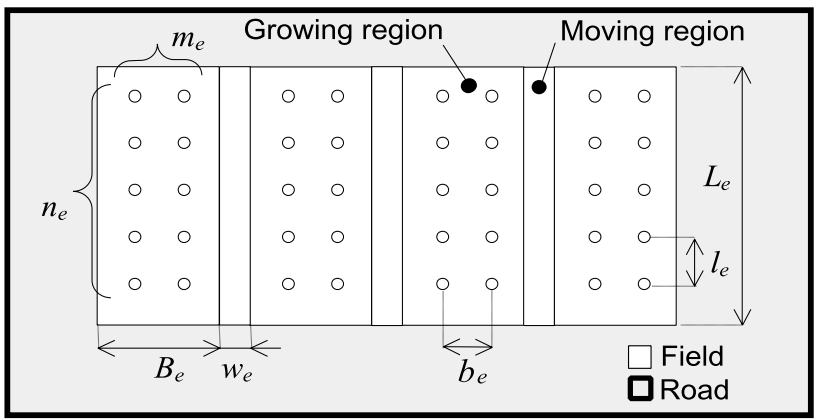

Fig. 2 A working environmental model for agricultural robots

plants (not including the target plants). Degree of danger (Kondo and Ting 1998) is used for collision avoidance between agricultural robots and humans. Nevertheless, these indexes evaluate only local performances in both space and time. From the viewpoint of holistic study of agricultural robots, there is a potentially useful global index, called the "theoretical field capacity" (TFC), which has been applied to existing agricultural vehicles, such as tractors, transplanters, and harvesters (e.g., Grisso et al. 2004). TFC is defined as follows,

$C_{t}=w_{m} V_{m}$,

where $w_{m}$ is the machine working width and $V_{m}$ is the machine straight running velocity. Actually, $w_{m}$ is the width of the end-effector and $V_{m}$ is defined as the maximum velocity with sufficient working performance. In this section, the subscript $m$ implies a dependence on only working machine, the subscript $e$ implies a dependence on only working environment, and the subscript $c$ implies a dependence on both.

TFC does have two problems. First, TFC cannot be used for high-degree-of-freedom mechanisms such as manipulators because it is based on the end-effectors of existing agricultural vehicles, that is, low-degree-of-freedom mechanisms. Second, TFC does not consider turning and (un)loading nor any interactions between agricultural robots and their working environments.

In order to solve these problems, we introduce a global performance index for agricultural robots. First, we start with a working environmental model. In the field of agriculture, working environments are classified into two subenvironments, namely, fields and roads. These imply that locomotion is also classifiable into "locomotion within a field" and "locomotion between fields (locomotion on roads)." Figure 2 shows a working environmental model for agricultural robots. The field consists of growing regions where plants (lattice points) grow and moving regions where robots move, and where $B_{e}$ : width of the growing region, $w_{e}$ : width of the moving region, $L_{e}$ : length of both the growing and moving regions, $m_{e}$ : column number of the lattice points, $n_{e}$ : row number of the lattice points, $b_{e}$ : interval between 
lattice points in the width direction, $l_{e}$ : interval between lattice points and the distance from the upper (or lower) sides of the growing region to the closest lattice point in the length direction.

This model has the following properties:

$L_{e} \geq B_{e} \geq w_{e}$,

$n_{e} \gg m_{e}$

and the plant density is defined and approximated as follows:

$\rho_{e}=\frac{n_{e} m_{e}}{B_{e} L_{e}}=\frac{n_{e} m_{e}}{B_{e}\left(n_{e}+1\right) l_{e}} \approx \frac{m_{e}}{B_{e} l_{e}}$.

This approximation is linked to the economy of time and effort of $n_{e}$ measurements.

Let $S_{e}$ be the field area and constant, then the number of growing regions is given by

$N_{e}=\frac{S_{e} / L_{e}-B_{e}}{B_{e}+w_{e}}+1$,

which is a global working space equation.

On the other hand, let $M_{c}$ be the number of growing regions where the robot simultaneously perceives and manipulates, that is, the number of growing regions in the intersection of the workspace and the field of view. For conventional agricultural vehicles, $w_{m}=M_{c} B_{e}$ holds. By considering a normative task plan (see the Appendix or Sakai et al. 2004 for details), then the robot's working time of straight running in $M_{c}$ growing regions is as follows

$$
\begin{aligned}
T_{c}^{s}= & \rho_{e} M_{c} B_{e} L_{e}\left(T_{m}^{m}+T_{m}^{p}\right)+\frac{\rho_{e} M_{c} B_{e} L_{e}}{n_{c}} T_{c}^{l} \\
& +\frac{L_{e}+L_{c}^{l}}{V_{m}}
\end{aligned}
$$

where $T_{m}^{m}$ : manipulation time for a plant, $T_{m}^{p}$ : perception time for a plant, $T_{c}^{l}$ : (un)loading time for the loading capacity of $n_{c}$ plants, $L_{c}^{l}$ : distance for (un)loading and $O\left(\frac{\rho_{e} M_{c} B_{e} L_{e}}{2 n_{c}} t\right) L_{e}$. The third term of $T_{c}^{s}$ is the time for the robot to move from the lower (upper) side of the moving region to the upper (lower) side while shuttling back and forward for (un)loading at the sides. The working time of turning for $M_{c}$ growing regions is

$T_{c}^{t}=T_{m}^{\pi}+\frac{M_{c}\left(B_{e}+w_{e}\right)}{V_{m}}$,

where $T_{m}^{\pi}$ is the 180-degree rotation time. In all, the total working time is

$T_{c}=\frac{N_{e}}{M_{c}}\left(T_{c}^{s}+T_{c}^{t}\right)$, which is a global working time equation. The symbols for the ceiling or floor functions are omitted for simplicity.

From (5) and (8), a new evaluation index is proposed as

$C_{e t}=\frac{\rho_{e} N_{e} B_{e} L_{e}}{T_{c}}$,

which is a working frequency that is well-defined for highdegree-of-freedom mechanisms in the first problem of TFC (i.e., TFC cannot be used for high-degree-of-freedom mechanisms). In the case of existing agricultural vehicles, if $w_{e}=0$,

$C_{e t} \rightarrow \rho_{e}\left(M_{c} B_{e} V_{m}\right)=\rho_{e} C_{t}$,

as $T_{m}^{m}+T_{m}^{p}, T_{c}^{t} \rightarrow 0$ and $n_{c} \rightarrow \infty$. This corresponds to the second problem of TFC (i.e., TFC does not take turning and (un)loading into account and does not consider interactions between agricultural robots and the working environment). In (10), $C_{e t}$ is an extension of $C_{t}$ (an extended theoretical field capacity).

\section{Structural systems}

In this section, we discuss the selection and design of structural systems for the mobile platform and manipulator with a hand.

\subsection{Design strategy of agricultural robots}

The design strategies of agricultural robots with locomotion and manipulation are classified as follows:

(AR1) Selection of the mobile platform and the existing stationary manipulator, and then the superposition of them

(AR2) Simultaneous design of the mobile mechanism and the manipulator

(AR3) Selection of the mobile platform, and then the design of only the manipulator

(AR4) Selection of the stationary manipulator, and then the design of only the mobile mechanism

(AR1) is the initial cost-emphasized case, (AR2) is the performance-emphasized case, (AR3) and (AR4) are the intermediate cases. We select (AR3) because both the initial cost and performance cannot be neglected in the field of agriculture and because there are many mobile platforms for existing agricultural vehicles. According to (AR3), first, we select a mobile platform from existing ones and, second, we design a manipulator that is suitable to not only the intended task but also to the selected mobile platform. 
Table 1 Parameters of the working environmental model (watermelon field)

\begin{tabular}{|c|c|c|c|c|c|c|}
\hline Place & $\begin{array}{l}\text { Yamagata } \\
\text { Obanazawa }\end{array}$ & $\begin{array}{l}\text { Chiba } \\
\text { Tomisato }\end{array}$ & $\begin{array}{l}\text { Nagano } \\
\text { Matsumoto }\end{array}$ & $\begin{array}{l}\text { Fukui } \\
\text { Sakai }\end{array}$ & $\begin{array}{l}\text { Tottori } \\
\text { Fukube }\end{array}$ & $\begin{array}{l}\text { Kumamoto } \\
\text { Kamoto }\end{array}$ \\
\hline Date & 2000.8 & 2000.6 & 1999.8 & 2000.5 & 2000.8 & 2000.5 \\
\hline$B_{e}[\mathrm{~m}]$ & 5.0 & 1.8 & 3.6 & 10.0 & 3.3 & 2.5 \\
\hline$w_{e}[\mathrm{~m}]$ & 0.5 & 0.8 & 1.6 & 1.4 & 0.8 & 0.5 \\
\hline$l_{e}[\mathrm{~m}]$ & 0.7 & 0.8 & 0.8 & 0.8 & 0.8 & 0.5 \\
\hline$m_{e}$ & 1 & 1 & 2 & 2 & 1 & 1 \\
\hline
\end{tabular}

\subsection{Task}

Here, the task of the heavy material handling agricultural robot is to harvest watermelons. Edan et al. (2000) developed a melon-harvesting robot. Hwang and Kim (2003) developed a watermelon-harvesting robot with a tele-operation system. Tokunaga et al. (1999) developed a digital circuit vision system for a watermelon-harvesting robot. These studies mainly address only the structural system or control system. In this present paper, we discuss both simultaneously.

Table 1 shows the environmental parameters investigated at some of the main product districts in Japan. The conventional operating procedure for watermelon harvesting was also investigated and divided into the following four steps.

STEP 1: Select targeted watermelons and cut the vines STEP 2: Pick them and place them on a delivery vehicle STEP 3: Drive the vehicle and reload them onto a truck STEP 4: Drive the truck and unload them at a factory

STEP 1 does not require hard labor. For STEP 3 and STEP 4, working machines (e.g., vehicles with lifter) have already been developed since these operations require hard labor.

For STEP 2, however, working machines have not yet been developed, even though STEP 2 requires hard labor. Picking watermelons requires the workers to have a high endpoint force. Various obstacles such as leaves, vines and unselected watermelons constrain the workers' orientation. More precisely, the workers are required to handle 6-12 kg watermelons while standing on tiptoe. This implies that STEP 2 requires the workers to generate a high joint torque. To summarize, STEP 2 is a challenging task.

Our ultimate goal is the robotization of STEP 2. The task of robotic STEP 2 operations is as follows.

TASK 2A: Perceive the watermelons from a far distance

TASK 2B: Locomote to their neighborhood

TASK 2C: Perceive them from a close distance

TASK 2D: Manipulate (pick and place) them

After completing TASK 2D, TASK 2A or TASK 2C follows.

In this study, TASK 2D is realized and evaluated. See Sakai et al. (2005) regarding the realization of TASK 2C. It should be noted that skilled workers persistently complete
Table 2 Classification of agricultural mobile mechanism

\begin{tabular}{lll}
\hline & $\begin{array}{l}\text { A-type: } \\
\text { supported by multiple } \\
\text { moving regions }\end{array}$ & $\begin{array}{l}\text { Y-type: } \\
\text { supported by single } \\
\text { moving region }\end{array}$ \\
\hline $\begin{array}{l}\text { Wheel type } \\
\text { Crawler type }\end{array}$ & Selected \\
Leg type & \\
Snake-like type & \\
\hline
\end{tabular}

the operation of picking one watermelon in about $10 \mathrm{~s}$ on average.

\subsection{Mobile platform selection}

Land mobile platforms are classified as shown in the rows of Table 2. Although the leg type and the snake-like type are still in the research and development stage, the wheel type and the crawler type are in the commercialization stage. As mentioned in Sect. 3.1, numerous mobile platforms exist for agricultural vehicles. The crawler type presents advantages in locomotion "within a field," because the bearing capacity of soil within a field is lower than that "between fields." The wheel type presents advantages in locomotion between fields in terms of speed.

We selected the crawler type, since the heavy material handling robot must locomote within a field even when it is raining or immediately after raining, that is, even when the bearing capacity becomes too low. For locomotion between fields, transportation by another wheel-type platform, such as a truck, is assumed and this is discussed later in this subsection.

Agricultural mobile platforms are classified as shown in the columns of Table 2. The A-type "strides over" growing regions while forming an A-like figure. The Y-type does "not stride over" any growing region (and may have a counterweight) while forming a Y-like figure. See the Appendix for examples.

The A-type has one advantage, namely, falling stability since the A-type satisfies $w_{m} \geq B_{e}$. Nevertheless, we selected the Y-type because the A-type is not suitable to transportation by another wheel-type platform, especially in the 
Table 3 Link length and link length ratio

\begin{tabular}{lllllllll}
\hline & $l_{0}$ & $l_{1}$ & $l_{2}$ & $l_{3}$ & $d_{1 \max }$ & $d_{2 \max }$ & $d_{3 \max }$ & $1-r_{l}: r_{l}$ \\
\hline Parallel & 0.5 & 0.5 & $(2.0)$ & 0.0 & - & - & $(5.0)$ & $d_{3 \max }: l_{2}$ \\
Polar & 0.5 & 0.5 & - & - & - & - & 5.0 & - \\
Articulated & 0.5 & 0.5 & $(3.0)$ & $(2.0)$ & - & - & - & $l_{2}: l_{3}$ \\
Cylindrical & 0.5 & - & - & - & - & $(2.0)$ & $(3.0)$ & $d_{2 \max }: d_{3 \max }$ \\
Cartesian & - & - & - & - & $(2.0)$ & 2.0 & $(2.0)$ & $d_{1 \max }: d_{3 \max }$ \\
SCARA & 0.5 & 0.5 & $(2.75)$ & $(2.75)$ & - & - & 1.0 & $l_{2}: l_{3}$ \\
\hline
\end{tabular}

case of large $B_{e}$, such as the watermelon cases shown in Table 1 .

Now we select a commercial self-propelled crawler vehicle $(145 \mathrm{~kg})$ with an engine $(3.2 \mathrm{~kW})$ for TASK $2 \mathrm{~B}$, as one candidate for the Y-type crawler platform.

From (5), $N_{e}$ decreases as $w_{e}$ increases. This becomes a more serious problem when $S_{e}$ is large. The vehicle width is $490 \mathrm{~mm}$, which is sufficiently small to pass through the narrow width $w_{e}$ and can keep the existing $w_{e}$ in Table 1 . While $C_{t}$ does not depend on $w_{e}$, we can see that a small value of $w_{e}$ can increase the numerator of $C_{e t}$, which can be considered as an index of the expected sales.

\subsection{Manipulator design (kinematics)}

Many well-known kinematic models exist, such as the polar coordinate type, articulated type, cylindrical coordinate type, Cartesian coordinate type, and SCARA type which was proposed from a practical viewpoint for assembly operations in industrial settings.

The design guidelines of a kinematic model of the heavy material handling manipulator are summarized below:

[G1] High normalized endpoint force in the vertical direction

[G2] High normalized workspace volume

[G3] High suitability to a mobile platform

[G4] High performance of contact safety

[G5] Low initial cost

where [G3] is introduced because (AR3) is selected.

In this study, from a practical viewpoint similar to that for SCARA type, the candidate proposed for heavy material handling in agriculture is shown in Fig. 3(a). This candidate has four degrees of freedom (4 DOF) and is termed the "parallel type."

This type is generalized from the original design (Umeda et al. 1997), but it can achieve a higher falling stability by removing the triangular geometry constraint. Other wellknown types are also shown in Fig. 3.

First, we determine that the endpoint position configuration (the three joints configuration) is designed to satisfy [G1][G2][G3] by performing a kinematics analysis for the numerical example given in Table 3. Second, we determine that the endpoint orientation configuration (an additional joint configuration) is designed to satisfy [G4][G5]. This process is discussed later in this section.

The direct kinematics of the rigid manipulator is given by

$x=r(q)$,

where $x$ is the endpoint position and $q$ is the joint displacement. For the parallel type, the kinematics are as follows:

$r(q)=\left[\begin{array}{c}\left(l_{2} \cos \theta_{2}+d_{3}\right) \cos \theta_{1} \\ \left(l_{2} \cos \theta_{2}+d_{3}\right) \sin \theta_{1} \\ l_{0}+l_{1}+l_{2} \sin \theta_{2}+l_{3}\end{array}\right]$,

where $q=\left[\begin{array}{lll}\theta_{1} & \theta_{2} & d_{3}\end{array}\right]^{T}$. Details about the other types are not described here since they are well known.

\subsubsection{Endpoint force}

Manipulability ellipsoids are represented by the following equation

$\dot{x} \in \operatorname{Im}(J(q)) \quad$ s.t. $\quad \dot{x}^{T} J(q)^{-T} J(q)^{-1} \dot{x} \leq 1$,

where $\operatorname{Im}(\bullet)$ is the image space and $J(q)=\partial r / \partial q \in \mathrm{R}^{3 \times 3}$ is a Jacobian.

Figure 4 shows the manipulability ellipsoids that are normalized by the summation $L$ of each link length in Table 3 . Note that the endpoint force is large in the direction where the manipulability is small because the endpoint force is the dual of the manipulability (Yoshikawa 1985). The vertical endpoint force of the polar coordinate type and that of the articulated type tend to be smaller than that of the parallel type. Especially when targets exist on ground level, such as in heavy vegetable harvesting, the vertical endpoint force on the ground of the polar coordinate type and the articulated type decrease as the endpoint position leaves the base. These imply that the polar coordinate type and the articulated type do not satisfy [G1]. 


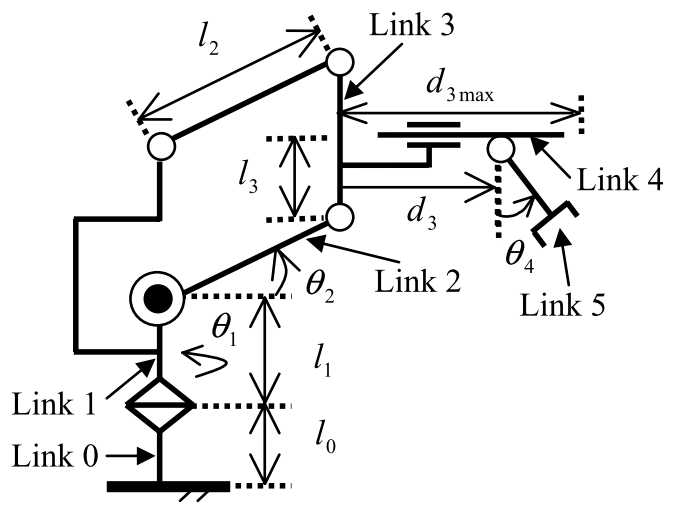

(a) Parallel type

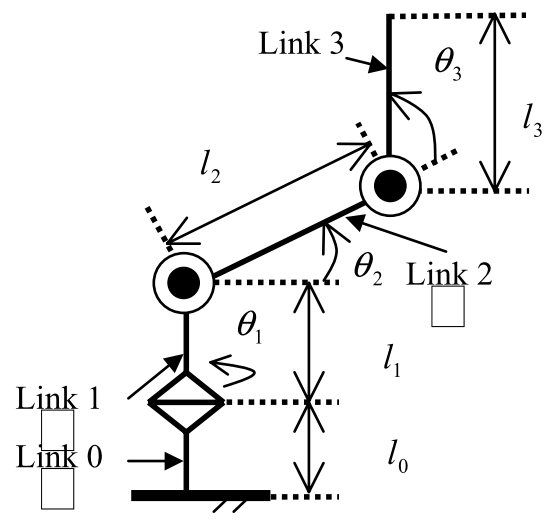

(c) Articulated type

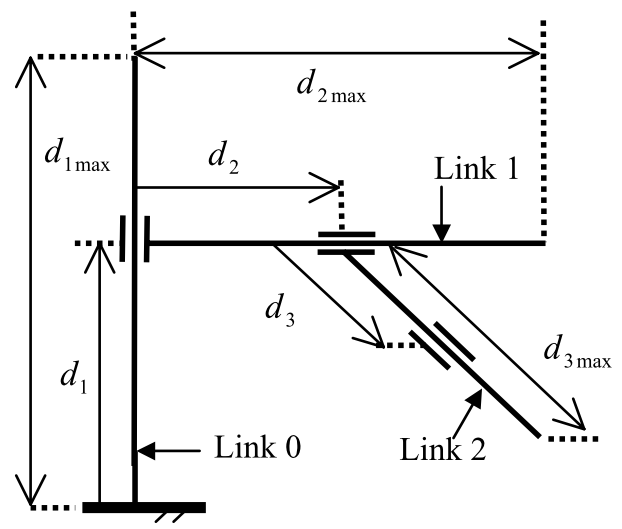

(e) Cartecian coordinates type

Fig. 3 Kinematic models

\subsubsection{Workspace volume}

Normalized workspace volume (Yang and Lee 1984) is expressed as

$V_{N}=\int_{V} d V / \frac{4}{3} \pi L^{3}$

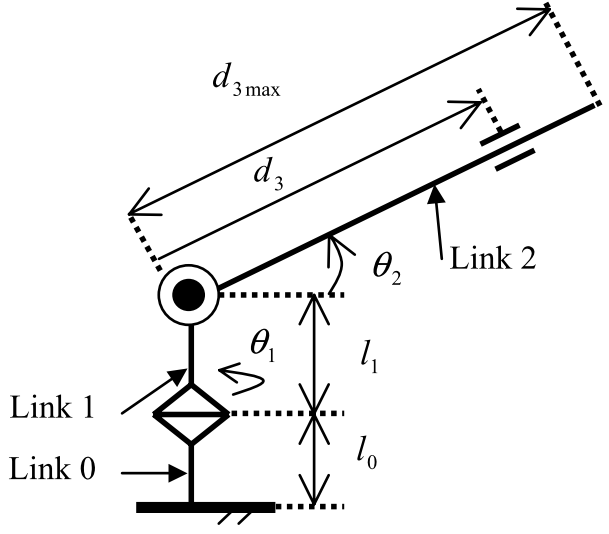

(b) Polar coordinates type

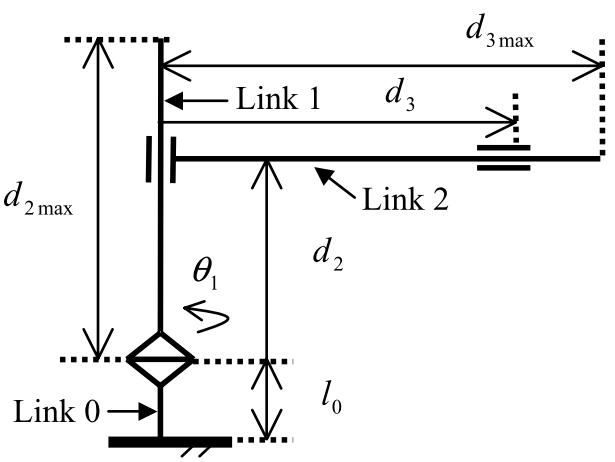

(d) Cylindrical coordinates type

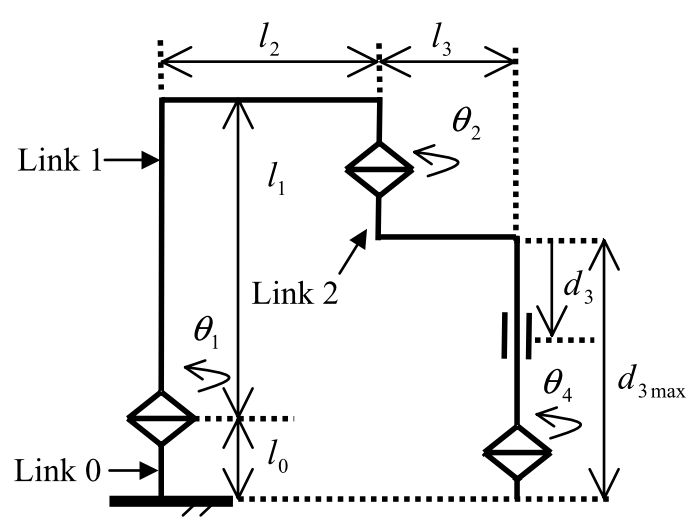

(f) SCARA type

where the integral sign denotes a volume integral.

Figure 5 shows the relationship between the link length ratio $r_{l}$ and the normalized workspace volume. Each link length ratio $r_{l}$ is defined as in Table 3 . The respective normalized workspace volumes of the cylindrical coordinate type, the Cartesian coordinate type and the SCARA type are smaller than that of the parallel type, irrespective of their 


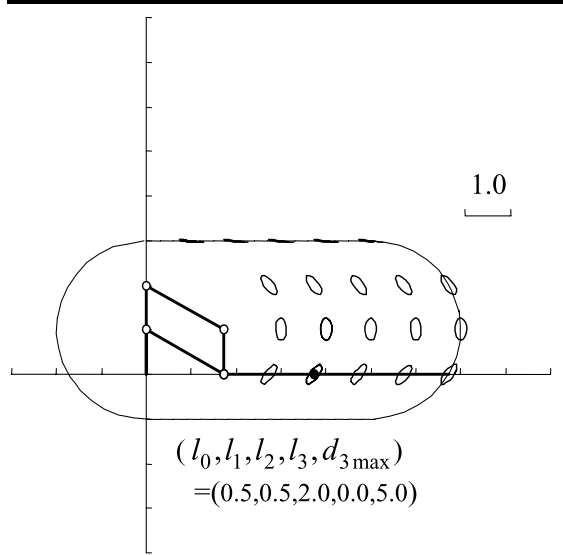

(a) Parallel type

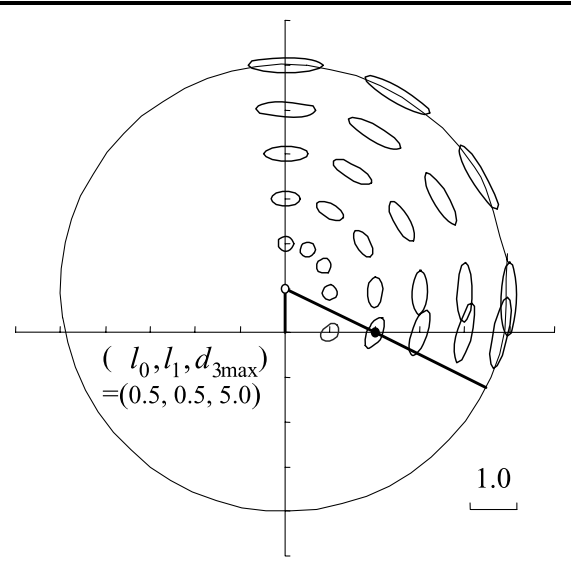

(b) Polar coordinates type

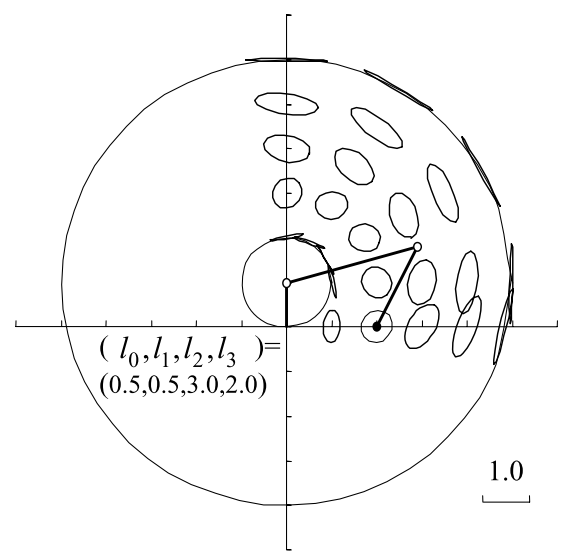

(c) Articulated type

Fig. 4 Normalized manipulability

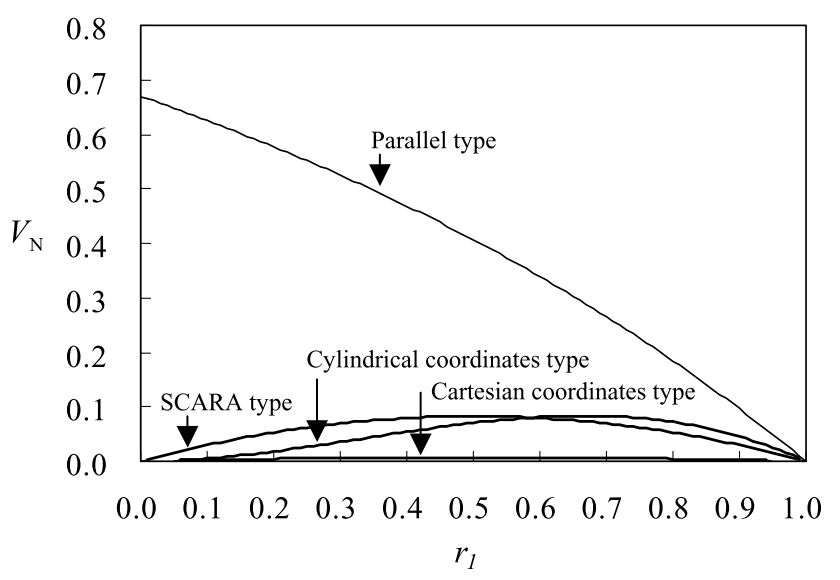

Fig. 5 Normalized workspace volume

link length ratio. This fact implies that the cylindrical coordinate type, the Cartesian coordinate type and the SCARA type do not satisfy [G2].

\subsubsection{Suitability to a mobile platform}

In general, as the normalized workspace volume increases, the required moving distance decreases. Figure 5 shows that the suitabilities to a mobile platform of the cylindrical coordinate type, the Cartesian coordinate type and the SCARA type are lower than that of the parallel type.

Also, in general, as the manipulability becomes more independent of the endpoint position, the required control of the mobile platform becomes simpler. This is all the more important in the case of the crawler type because the crawler type has no omnidirectional characteristic. Note that we do not discuss the other types, such as the wheel type, at this time, because we already selected the crawler type as our mobile platform in Sect. 3.3 under the guideline (AR3) given in Sect. 3.1. The manipulability (and the vertical endpoint force) of the parallel type is completely constant on ground level where the targets are located in the case of heavy vegetable harvesting. Figure 4 implies that the suitabilities to a mobile platform of the polar coordinate type and the articulated type is lower than that of the parallel type.

Now, we can conclude that the parallel type best satisfies [G1][G2][G3]. In the analysis in the remainder of this section, we check whether the parallel type satisfies [G4] and [G5] or not.

\subsubsection{Performance of contact safety and initial cost}

In the fields of welfare and human care, safety strategies for collisions between robots and humans are classified into control strategies and machine design strategies, according to the situations and actions before and after a collision (Ikuta and Notaka 1999). From the viewpoint of [G4], we apply this classification to a safety strategy for the collision between a robot and an agricultural target (e.g., fruit, eggs and cow udders). We select a control strategy for before a collision and a machine design strategy for after a collision.

More precisely, a control system is designed to achieve small positional errors to avoid undesirable collisions and an additional joint (Joint 4) is designed to be passive to reduce the contact force immediately after a collision. This machine design strategy has an additional advantage from the viewpoint of [G5]. The passive joint results not only in an actuator reduction but also in an inclination sensor reduction because the steady state of the Joint 4 displacement is always equal to the inclination angle, as a result of the kinematics shown in Fig. 3(a). If we select a control strategy after a collision, we might require force sensors, and have more difficulty in satisfying [G5].

\subsection{Manipulator design (structure)}

Figure 6 shows a schematic diagram of the manipulator. From (9), $C_{e t}$ decreases as $M_{c}$ increases, and this is more ef- 


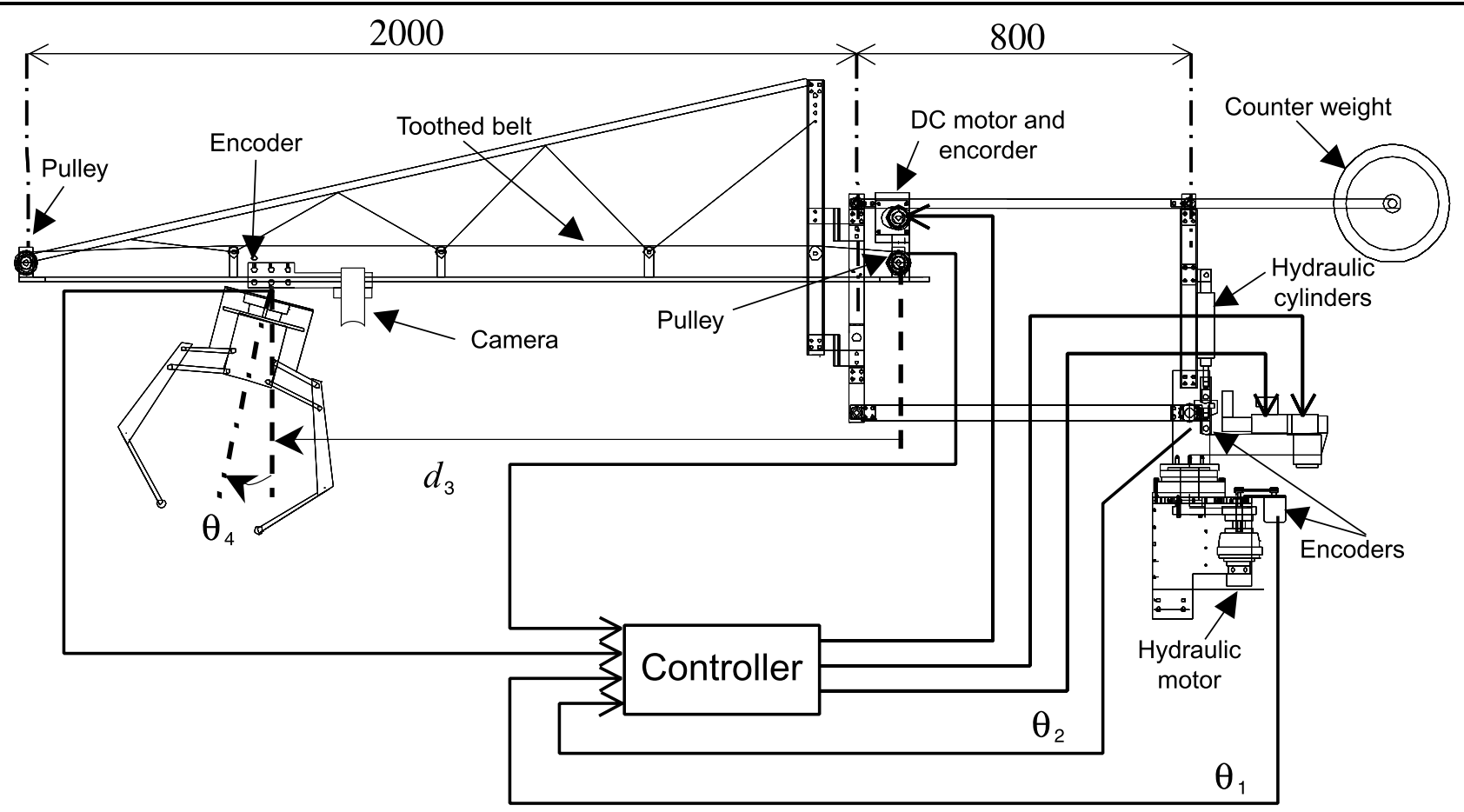

Fig. 6 Schematic diagram of the manipulator

fective when $T_{m}^{\pi}$ and $L_{e}$ are large or $V_{m}$ is small. The maximum horizontal reach of the manipulator is $2.8 \mathrm{~m}$, which is sufficiently long to make $M_{c}$ large, especially for small $B_{e}$, and to keep the existing $B_{e}$ in Table 1. While $C_{t}$ is illdefined for robot manipulators, we can see that large $M_{c}$ by long manipulators increases the global performance of $C_{e t}$.

\subsubsection{Transducer \& power source selection and transmission \& circuit design}

Three joints (Joint 1, Joint 2 and Joint 3 ) are active joints. A hydraulic motor actuates Joint 1 . The torque of the motor is transferred to the harmonic drive gear $(1 / 50)$ through a toothed belt and two pulleys. Two hydraulic cylinders actuate Joint 2. The force of the cylinders is converted through a mechanical linkage, and the maximum vertical endpoint force is $150 \mathrm{~N}$. The hydraulic actuators have direct-type servo valve drivers.

The power source of the hydraulic actuators is the crawler vehicle engine. From the viewpoint of [G3], the hydraulic system is advantageous since only a pulley and a belt are required for conversion from an engine $(3.2 \mathrm{~kW}, 2000 \mathrm{rpm})$ to a pump $(0.2 \mathrm{l} / \mathrm{s})$.

A DC motor $(110 \mathrm{~W})$ with a reduction gear $(1 / 15)$ actuates Joint 3 . The rotary motion of the motor is converted to translational motion through a toothed belt, two toothed pulleys, and a slider. The slider has four small bearings as wheels so that it can move on the rail. The DC motor has a current driver. The power source is a battery $(24 \mathrm{~V})$ and a vehicular generator. Unlike hydraulic cables, electrical power cables can be easily disconnected. Therefore, this long manipulator also can be disconnected for transportation by a truck.

Joint 4 consists of a bearing attached to the slider without dampers. From the viewpoint of high-speed operation, viscous friction is preferable for reduction of the remaining oscillation of Joint 4. However, from the viewpoint of [G4] (the safety strategy after collision), viscous friction is not preferable. That is, viscous friction increases the contact force on the target and the environment. This trade-off problem cannot be solved by using only machine design, such as a damper attachment. As described later, switching and scheduling of controllers can be used to solve this trade-off problem.

Figure 7 shows the drive (hydraulic and electric) circuits. The controller consists of a D/A converter (12-bit), a digital computer $(25 \mathrm{MHz})$ and a counter board (24-bit). Optical rotary encoders $(1024,1024,1000,200 \times 24 \mathrm{P} / \mathrm{R})$ measure the displacement of all joints. All inputs $\left(v_{1}, v_{2}, v_{3}\right)$ are constrained by $\pm 5 \mathrm{~V}$ and all outputs $\left(\theta_{1}, \theta_{2}, d_{3}, \theta_{4}\right)$ are constrained as shown in Table 4. The manipulator is a 3-input 4-output system.

\subsubsection{End-effector selection and redesign}

We select a hand and redesign it for picking and placing watermelons. From the viewpoint of [G5], we select an orig- 


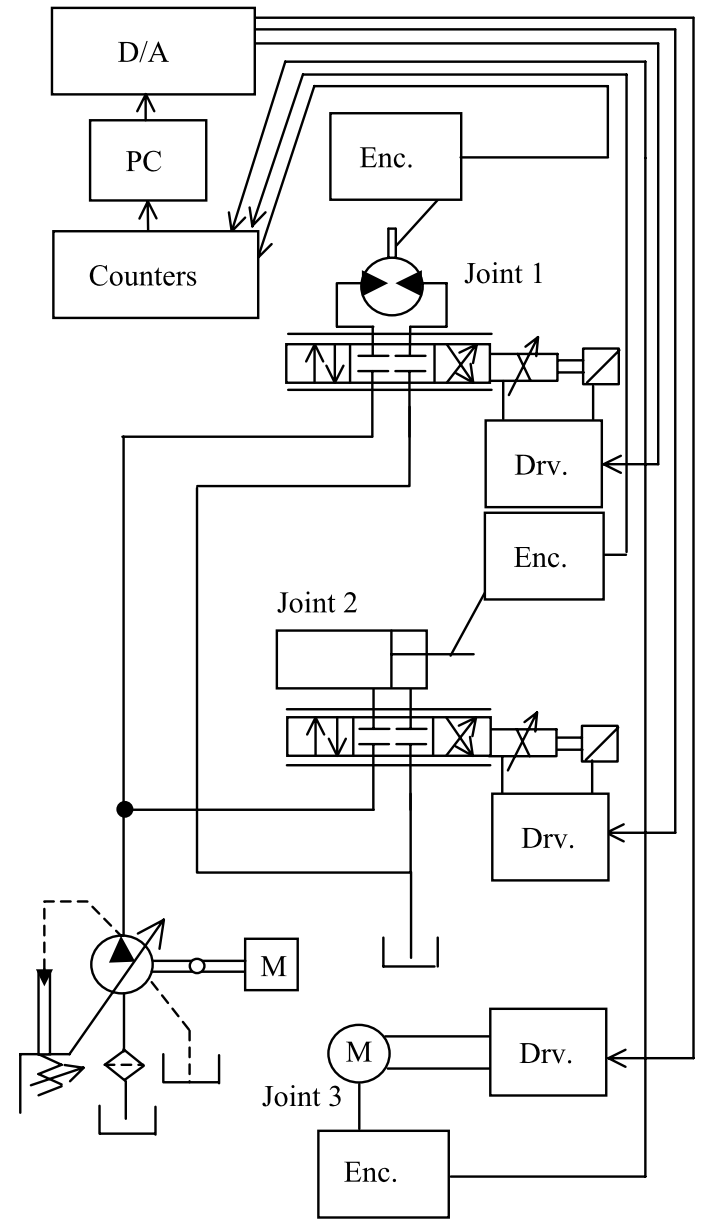

Fig. 7 Drive circuit

Table 4 Robot specifications

\begin{tabular}{lll}
\hline Vehicle & Mass & $145[\mathrm{~kg}]$ \\
& Width & $490[\mathrm{~mm}]$ \\
\hline Manipulator & Mass & $90[\mathrm{~kg}]$ \\
& Operating range & $-115<\theta_{1}<+115[\mathrm{deg}]$ \\
& $-25<\theta_{2}<+25[\mathrm{deg}]$ \\
& $0<d_{3}<1890[\mathrm{~mm}]$ \\
& $-30<\theta_{4}<+30[\mathrm{deg}]$ \\
& $2800[\mathrm{~mm}]$
\end{tabular}

inal type of hand (Iida et al. 1995) consisting of four 1DOF fingers with no actuators. It performs picking using passive force closure within a $40-\mathrm{mm}$ allowable positional error. However, this hand cannot perform placing without a hydraulic actuator.

We focus on the fact that reaction forces are transmitted from the platform to the finger during placing. We design a wire mechanism to realize placing using less power, as shown in Fig. 8. First, the wires suspend the fingers and

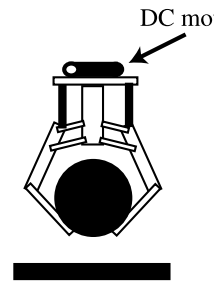

(a)

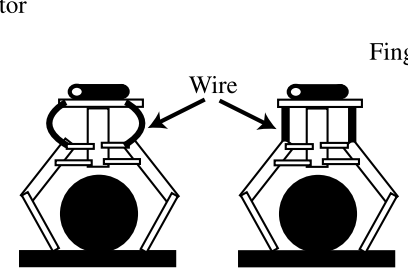

(b)

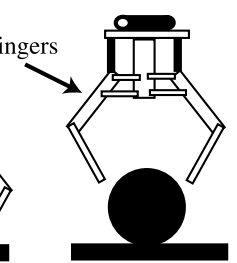

(d)
Fig. 8 Motion plan for placing using reaction forces

gravity keeps the finger closed (Fig. 8(a)). Second, after the hand contacts the platform by the Joint 2 motion, the wire tension can be zero because of the reaction forces (Fig. 8(b)). Third, a low-power DC motor reels the wires and keeps the fingers open (Fig. 8(c)). Finally, the hand rises without recontacting the objects (Fig. 8(d)).

\section{Control systems}

This section discusses a control system designed in an analytical way. We design controllers experimentally without considering robustness and then demonstrate the robust stability of the closed-loop system in the presence of parametric perturbation and uncertainty.

\subsection{Motion planning and controller design guideline}

The targeted watermelon is manipulated as follows.

M2D-1: The hand is moved above the watermelon by all active joints.

M2D-2: The hand picks the watermelon up with Joints 2 and 3.

M2D-3: The hand is moved above the platform with Joints 1 and 3 .

M2D-4: The hand places the watermelon with Joints 2 and 3.

Immediately after M2D-4, either TASK 2A or TASK 2C follows.

In this paper, we select PTP control to realize this motion plan and design the controllers in the next section. Here, the following uncertainty is expected in the manipulator.

- Parameter perturbations

The watermelon mass is $6-12.0 \mathrm{~kg}$. This mass can cause additional perturbations, such as changes in joint friction or in the moment of inertia, in addition to the original perturbations, which exist in the torque constant value and the joint friction.

- Unmodeled dynamics

In general, unmodeled dynamics exist for hydraulic systems because physical modeling of hydraulic systems is difficult. 
Table 5 Identified parameters

\begin{tabular}{llll}
\hline Link4 & Nominal & Maximum & Minimum \\
\hline$m[\mathrm{~kg}]$ & 40.31 & 62.58 & 18.04 \\
$c_{m}[\mathrm{Ns} / \mathrm{m}]$ & 90.18 & 135.51 & 44.84 \\
Link5 & Nominal & Maximum & Minimum \\
\hline$M[\mathrm{~kg}]$ & 4.06 & $(16.06)$ & $(4.06)$ \\
$c_{M}[\mathrm{Ns} / \mathrm{m}]$ & 0.0015 & - & - \\
$J\left[\mathrm{kgm}{ }^{2}\right]$ & 0.14 & - & - \\
$l[\mathrm{~m}]$ & 0.20 & - & - \\
\hline
\end{tabular}

However, some types of robust controllers (e.g., $H_{\infty}$ controller) give conservative results even though they are generated by reasonable design procedures. To avoid this problem, we design robust controllers in an analytical way. First, we design controllers experimentally without considering robustness. Next, we demonstrate the robust stability of the closed-loop system in the presence of parametric perturbation and uncertainty.

Furthermore, we separate the entire 3-input 4-output system into three subsystems: Joint 1 system, Joint 2 system, and Joint 3-Joint 4 system. Their mutual interferences are sufficiently weak, or at least do not influence the closed-loop stability at all (Sakai et al. 2007). First, we design the Joint 3-Joint 4 controller in the presence of parametric perturbation. Second, we design the Joint 1 controller and the Joint 2 controller in the presence of unmodeled dynamics.

\subsection{Modeling}

Equations of motion for the Joint 3-Joint 4 system are given as coupling between two rigid bodies.

$$
\begin{aligned}
& (m+M) \ddot{d}_{3}+M l \ddot{\theta_{4}} \cos \theta_{4}+c_{m} \dot{d}_{3}-M l \dot{\theta}_{4}^{2} \sin \theta_{4}=u_{3}, \\
& M l \ddot{d_{3}} \cos \theta_{4}+\left(J+M l^{2}\right) \ddot{\theta}_{4}+c_{M} \dot{\theta_{4}}+M g l \sin \theta_{4}=0,
\end{aligned}
$$

where $d_{3}$ : Joint 3 displacement [m] $\theta_{4}$ : Joint 4 displacement [rad] $m$ : Link 4 mass [kg] $M$ : Link 5 mass (the hand mass) [kg] $J$ : Link 5 moment of inertia about the center of mass $\left[\mathrm{kgm}^{2}\right] l$ : distance between the centers of mass $[\mathrm{m}] c_{m}$ : Link 4 viscous friction coefficient (Joint 3 friction coefficient) [Ns $/ \mathrm{m}] c_{M}$ : Link 5 viscous friction coefficient [Ns/rad] $g$ : acceleration due to gravity $\left[\mathrm{m} / \mathrm{s}^{2}\right] u$ : input force on Link $4[\mathrm{~N}]$. Table 5 lists the parameters identified. The transfer function from input $v_{3}$ to force $u_{3}$ is regarded as a static system with a gain 42.25 .

Around the unique stable equilibrium point, the statespace expression of the nominal linearized model is expressed as

$\left\{\begin{array}{l}\dot{x}=A x+B u_{3}, \\ y=C x+D u_{3},\end{array}\right.$ where $x=\left(\begin{array}{llll}d_{3} & \theta_{4} & \dot{d}_{3} & \dot{\theta}_{4}\end{array}\right)^{T}$ is the state, $y$ is the measured output,

$$
\begin{aligned}
& A=\left[\begin{array}{cccc}
0 & 0 & 1 & 0 \\
0 & 0 & 0 & 1 \\
0 & \frac{M^{2} l^{2} g}{N} & -c_{m} \frac{M l^{2}+J}{N} & \frac{c_{M} M l}{N} \\
0 & \frac{-(M+m) M g l}{N} & c_{m} \frac{M l}{N} & \frac{-c_{M}(M+m)}{N}
\end{array}\right], \\
& B=\left[\begin{array}{llll}
0 & 0 & \frac{M l^{2}+J}{N} & \frac{-M l}{N}
\end{array}\right]^{T}, \quad C=I_{4}, \quad D=0^{4 \times 1},
\end{aligned}
$$

and $N=(M+m) J+M m l^{2}$. The joint velocities are given by the first order difference of the joint displacement.

\subsection{Controller design}

For the Joint 3-Joint 4 system, we design a switched controller consisting of one PD controller $K_{1}$ and two LQ controllers $K_{2}$ and $K_{3}$, where $K_{1}$ controls only the Joint 3 displacement, $K_{2}$ and $K_{3}$ controls both the Joint 3 and Joint 4 displacement. We could find no single controller with sufficient performance because of the parametric perturbations. These three controllers are switched according to the endpoint position of the manipulator.

Integral action was not applied instead of switching due to the large input saturation at large initial values, which is an important problem associated with long and translational robotic arms in general. This is the reason why nonlinear friction (such as Coulomb and Stribeck) was not modeled explicitly in (15).

The two LQ controllers are designed for the linearized model and the following evaluation criteria are used:

$J_{L Q_{i}}=\int_{0}^{\infty}\left(x^{T} Q_{i} x+r_{i} u_{3}^{2}\right) d t, \quad i=1,2$,
$Q_{1}=\operatorname{diag}(25,80,0,0), \quad r_{1}=0.0001$,
$Q_{2}=\operatorname{diag}(1800,400,0,0), \quad r_{2}=0.002$.

The respective controllers are

$u_{3}=\left\{\begin{array}{l}-K_{1} x=-[1478.9,0.0,246.5,0.0] x, \\ -K_{2} x=-[948.7,-416.4,307.9,9.8] x, \\ -K_{3} x=-[500.0,-859.6,279.3,-21.7] x,\end{array}\right.$

where the gains of $K_{1}$ and their switching conditions are tuned experimentally.

For the Joint 1 and Joint 2 systems, joint-independent PID controllers are implemented as

$$
\begin{aligned}
& v_{1}=0.01 \theta_{1}+0.009 \int \theta_{1} d t, \\
& v_{2}=0.03 \theta_{2}
\end{aligned}
$$


4.4 Control system analysis

The perturbed parameters of the Joint 3-Joint 4 system are described as

$\left(\begin{array}{lll}M m c_{m}\end{array}\right)=\left(\begin{array}{lll}\bar{M} \bar{m} \bar{c}_{m}\end{array}\right)+\left(\begin{array}{lll}p_{M} \delta_{M} & p_{m} \delta_{m} & p_{c_{m}} \delta_{c_{m}}\end{array}\right)$,

where $\bar{M}, \bar{m}$ and $\bar{c}_{m}$ are the mean values, $p_{M}, p_{m}$ and $p_{c_{m}}$ are the margins (half of the differences between the maximum and the minimum), and $\delta_{M}, \delta_{m}$ and $\delta_{c_{m}}$ are the normalized perturbations which satisfy

$\left|\delta_{M}\right| \leq 1, \quad\left|\delta_{m}\right| \leq 1, \quad \delta_{c m} \mid \leq 1$

and they are assumed to be LTI systems. In addition, $J$ is assumed to be $J=\bar{J}+\left(M-\left(\bar{M}-p_{M}\right)\right) l^{2}$, where $\bar{J}$ is the nominal. Table 5 shows the identified parameter perturbations.

In the motion plan just mentioned, $\delta_{M}$ is regarded as a static system. The robust stability of the closed loop is analyzed in the presence of the perturbation of $m$ and $c_{m}$ under arbitrary fixed $M$.

However, (16) clarifies that the elements of the coefficient matrices $A$ and $B$ are nonlinear with respect to the physical parameters. This nonlinearity implies that the analyzed result can be very conservative when the uncertainty is expressed as affine perturbations with respect to the coefficient matrices, or as affine perturbations with respect to the coefficient of the characteristic equation for Kharitonov's theorem. Actually, these approaches cannot demonstrate closedloop stability for the case of Table 5.

Therefore, once we notice that the physical parameters are written in rational polynomial form in $A$ and $B,(16)$ can be rewritten in a descriptor form, as follows:

$E_{\text {des }} \dot{x}=A_{\text {des }} x$,

$E_{\text {des }}=N I_{4}, \quad A_{d e s}=N\left(A-B K_{i}\right), \quad i=1,2,3$.

In these equations, the elements of the coefficient matrices are polynomial in form.

In this case, the following relation holds (Kawanishi and Sugie 1995).

$$
\begin{aligned}
{\left[E_{\text {des }} A_{d e s}\right] } & =F_{u}\left(S\left(\left[\begin{array}{ll}
0 & I_{8} \\
P & P_{o}
\end{array}\right], \tilde{M}\right), \Delta_{34}\right) \\
& =F_{u}\left(\bar{M}, \Delta_{34}\right),
\end{aligned}
$$

where

$P=\operatorname{diag}\left(p_{m} I, p_{c_{m}}\right)$,

$P_{o}=\operatorname{diag}\left(m_{o} I, c_{m o}\right)$,

$\Delta_{34}=\operatorname{diag}\left(\delta_{m} I, \delta_{c_{m}}\right)$,

$F_{u}(\bullet, \bullet)$ is the upper linear fractional transformation, and $S(\bullet, \bullet)$ is the star product.

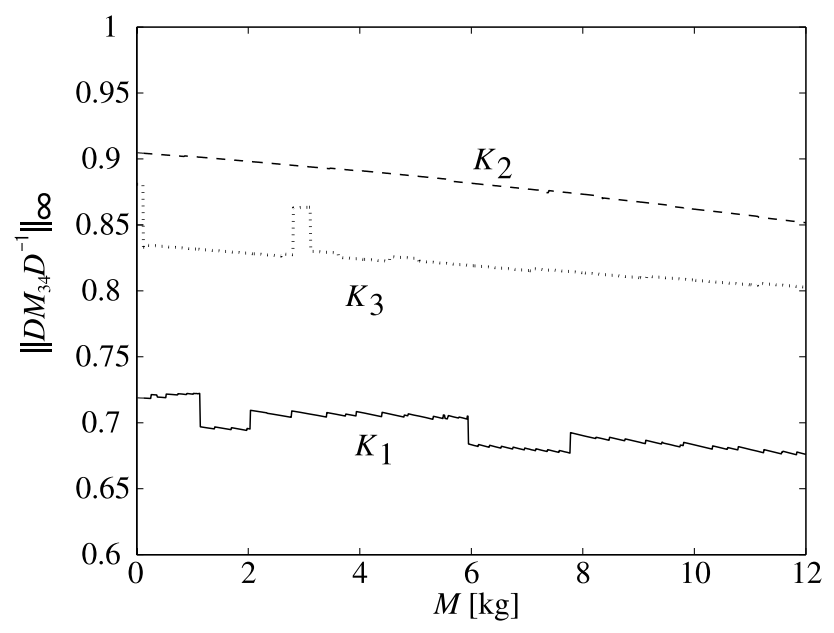

Fig. 9 Constant-scaled $H_{\infty}$ norm

Now, $\delta_{m}$ and $\delta_{c m}$ can be extracted successfully. By using Doyle's notation, (24) can be expressed as

$\left\{\begin{array}{l}z=M_{34} w=\left[\frac{E_{0}^{-1} A_{d e s} \mid E_{0}^{-1} \bar{M}_{21}}{-\bar{M}_{12 a} A_{0}+\bar{M}_{12 b} \mid \bar{M}_{11}-E_{0}^{-1} \bar{M}_{12 a} \bar{M}_{21}}\right] w, \\ w=\Delta_{34} z=\operatorname{diag}\left(\delta_{m} I, \delta_{c m} I\right) z,\end{array}\right.$

where $E_{0}$ and $A_{0}$ are the corresponding nominal values of $E_{d e s}$ and $A_{d e s}$ and

$\bar{M}=\left[\begin{array}{ll}\bar{M}_{11} & \bar{M}_{12} \\ \bar{M}_{21} & \bar{M}_{22}\end{array}\right]$,

$\bar{M}_{12}=\left[\begin{array}{ll}\bar{M}_{12 a} & \bar{M}_{12 b}\end{array}\right], \quad \bar{M}_{22}=\left[\begin{array}{ll}E_{0} & A_{0}\end{array}\right]$.

The dimensions and $\bar{M}$ are omitted because of their uniqueness.

According to the small gain theorem (Shamma 1994), under the set

$$
\begin{aligned}
D_{s}= & \left\{\operatorname{diag}\left(D_{1}, D_{2}\right) \text { s.t. } D_{1} \in \mathbf{C}^{7 \times 7},\right. \\
& \left.D_{2} \in \mathbf{C}^{1 \times 1}, D_{i}=D_{i}^{*}>0, i=1,2\right\} .
\end{aligned}
$$

A sufficient condition that the closed loop is stable for all $\Delta_{34}$ which satisfies $\left\|\Delta_{34}\right\|_{\infty} \leq 1$ is

$\exists D$ s.t. $\left\|D M_{34} D^{-1}\right\|_{\infty}<1, \quad D \in D_{s}$.

Figure 9 shows the constant scaled norm in (28) for the three designed controllers. This calculation is a feasible problem of linear matrix inequality (LMI) and can be solved using the MATLAB LMI Toolbox. The interval of $M$ is $0.01 \mathrm{~kg}$. Robust stability using the parameters in Table 5 is thus confirmed.

Next, we begin the analysis of the Joint 1 and Joint 2 systems. In addition to the above parametric perturbations, unmodeled dynamics should be taken into account because 
of the hydraulic actuators. As stated above, unmodeled dynamics generally exist for hydraulic systems because physical modeling of such systems is difficult.

A standard system identification experiment yields the nominal models as

$\theta_{1}(s)=P_{11} v_{1}(s)=\frac{640000}{s^{3}+160 s^{2}+6400 s} v_{1}(s)$,

$\theta_{2}(s)=P_{22} v_{2}(s)=\frac{25}{0.1667 s^{2}+s} v_{2}(s)$.

The corresponding additive uncertainty (Sakai et al. 2007) has upper bounds that are determined as

$W_{s 1}=\frac{0.068 s+1.7}{0.05 s+1}, \quad W_{s 2}=\frac{25 s+2500}{s+600}$.

Here, we call the structured singular value (Packard and Doyle 1993) as

$\mu_{\Delta}(M)=\frac{1}{\min \{\bar{\sigma}(\Delta) \mid \Delta \in \Delta, \operatorname{det}(I-M \Delta)=0\}}$,

unless no $\Delta \in \Delta$ renders $I-M \Delta$ as singular, in which case $\mu_{\Delta}:=0$. In our case,

$M=M_{12}=\operatorname{diag}\left(M_{1}, M_{2}\right), M_{i}=W_{s i} F_{l}\left(P_{i i}, K_{i i}\right)$,

$\Delta=\Delta_{12} \in\left\{\operatorname{diag}\left(\Delta_{1}, \Delta_{2}\right)\right.$ s.t. $\left.\Delta_{i} \in C\right\}, \quad i=1,2$,

where $F_{l}(\bullet, \bullet)$ is the lower linear fractional transformation and $\mu_{\Delta}\left(M_{12}\right) \leq 1$ is a sufficient condition for the closedloop stability for all $\Delta_{12}$ that satisfy $\left\|\Delta_{12}\right\|_{\infty} \leq 1$. In our case, the upper bound of $\mu$ is given as

$\sup _{w \in \boldsymbol{R}} \mu_{\Delta}=\max \left(\left\|M_{1}\right\|_{\infty},\left\|M_{2}\right\|_{\infty}\right)$

and the value is 0.751 (Matlab version 6.0). That is, robust stability is confirmed.

\section{Field experiments}

In this section, we evaluate the validity of the proposed structural system and its control system by performing watermelon harvesting experiments in an open field.

\subsection{Experimental methods}

We transplanted watermelon seedlings and grew them for four months. The growing method and the working environment were based on our investigation of the main watermelon-producing districts in Japan. The environmental parameters in Fig. 2 were selected as $\left(B_{e}, L_{e}, w_{e}, m_{e}\right)=$ $(2.5,10,0.5,1)$. The platform height of the conventional delivery vehicle was $820 \mathrm{~mm}$ for STEP 2 . The outdoor harvesting experiment was performed in August in Kyoto, Japan.

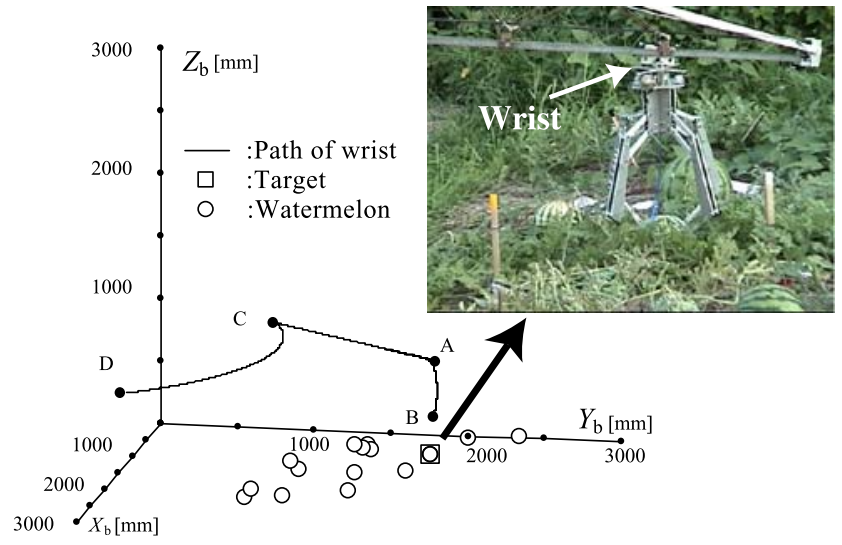

Fig. 10 Wrist path

This experiment assumed that TASK $2 \mathrm{C}$ had already been completed. That is, the positions of the targeted watermelon with no vines were given manually by an optical survey instrument. Validation of this assumption is given in a previous study by us (Sakai et al. 2005).

The desired joint displacements were determined by the watermelon positions in the base coordinate frame system and inverse kinematics. The base coordinate frame system was a right-hand system whose origin was at the intersection point of the road surface and the rotation axis of Joint 1 and whose $\mathrm{x}$-axis was parallel to the straight running direction. The equation of inverse kinematics was obtained explicitly from (12):

$q=\left[\begin{array}{c}\tan ^{-1}(y / x) \\ \sin ^{-1}\left(\left(z-l_{0}-l_{1}-l_{3}\right) / l_{2}\right) \\ \frac{x}{\cos \left(\tan ^{-1}(y / x)\right)}-l_{2} \cos \left(\sin ^{-1}\left(\frac{z-l_{0}-l_{1}-l_{3}}{l_{2}}\right)\right.\end{array}\right]$,

where $x \neq 0$ due to the Y-type mobile platform, which cannot have the target in the straight running direction from the robot base center.

Two experiments were performed. First, as a pilot experiment to confirm the validity of the structural system, switching was not applied and the controller was fixed to a PID control with low gains. Only picking was attempted. Second, to confirm the validity of the total system (both the structural system and the control system working together) with the proposed controllers, switching was applied and picking and placing were attempted.

In both experiments, we repeated this harvesting experiment 15 times at different watermelon positions. The displacement of Joint 4 was not controlled and the LQ controllers were not applied while grasping and releasing the watermelons to satisfy [G4]. The sampling time was $10 \mathrm{~ms}$.

\subsection{Experimental results}

Figure 10 shows the wrist path in the first experiment using low-gain PID control. In this figure, the solid line is the 


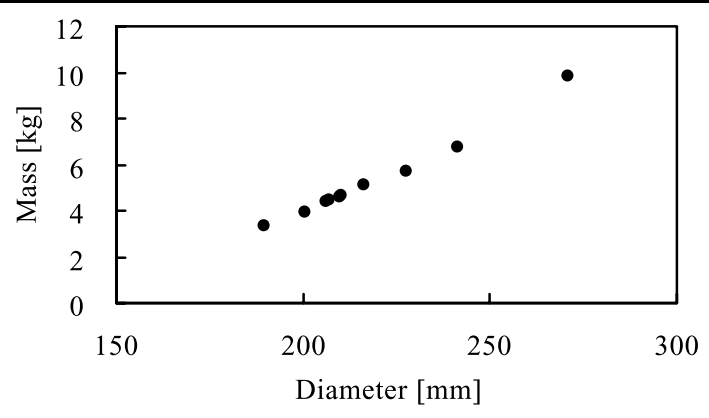

Fig. 11 Specifications of the watermelons

path of the wrist measured by the encoders, and the circles are the watermelon positions measured by the optical survey instrument.

In the first experiment, the success rate for robotic harvesting, defined as the percentage of successful operations to the total trial operations, was $86.7 \%$. There was no damage to the harvested watermelons. In the failure cases, in which the watermelons were not picked, the targeted watermelons were always the smallest ones and were more than $2 \mathrm{~m}$ from the base. The diameters and masses of these watermelons are shown in Fig. 11. The total operation time for one watermelon was $40 \mathrm{~s}$.

Figure 12 shows the harvest procedure and Fig. 13 shows the results of the joint displacements in the second experiment using the switched controller. This figure also shows the switching condition according to the endpoint position. The largest spikes of the Joint 2 displacement $\theta_{2}$ correspond to picking and the second-largest spikes correspond to the placing. The success rate was $86.7 \%$ and equal to the previous rate in the first experiment. There was no damage to the harvested watermelons. The total operation time was $14 \mathrm{~s}$.

\subsection{Discussion}

The success rate was greater than $66.7 \%$, which was reported in a previous study in our group (Iida et al. 1995). In the indoor laboratory experiments of the current study, the success rate was $100 \%$ for bowling balls and the positional error was $15 \mathrm{~mm}$, which is less than the allowable error of 40 $\mathrm{mm}$ given in Sect. 3.5.2. The failures seemed to be attributable to base motion due to the high angular momentum of the manipulator around Joint 1 , which is a property of PTP control. However, the failure watermelons were so small as to be out of the standard mass range $(6-12 \mathrm{~kg}$ ). These watermelons are important not during but only after the harvest season. The validity of the manipulator structure is thus confirmed.

For comparison, Fig. 13 also shows the case without LQ control. The controllers for Joints 1 and 2 are the same. It is clear that the Joint 4 displacement oscillates. When the Joint 2 displacement is a minimum in Fig. 13(b), the Joint 4 displacement without LQ control is much larger than that with

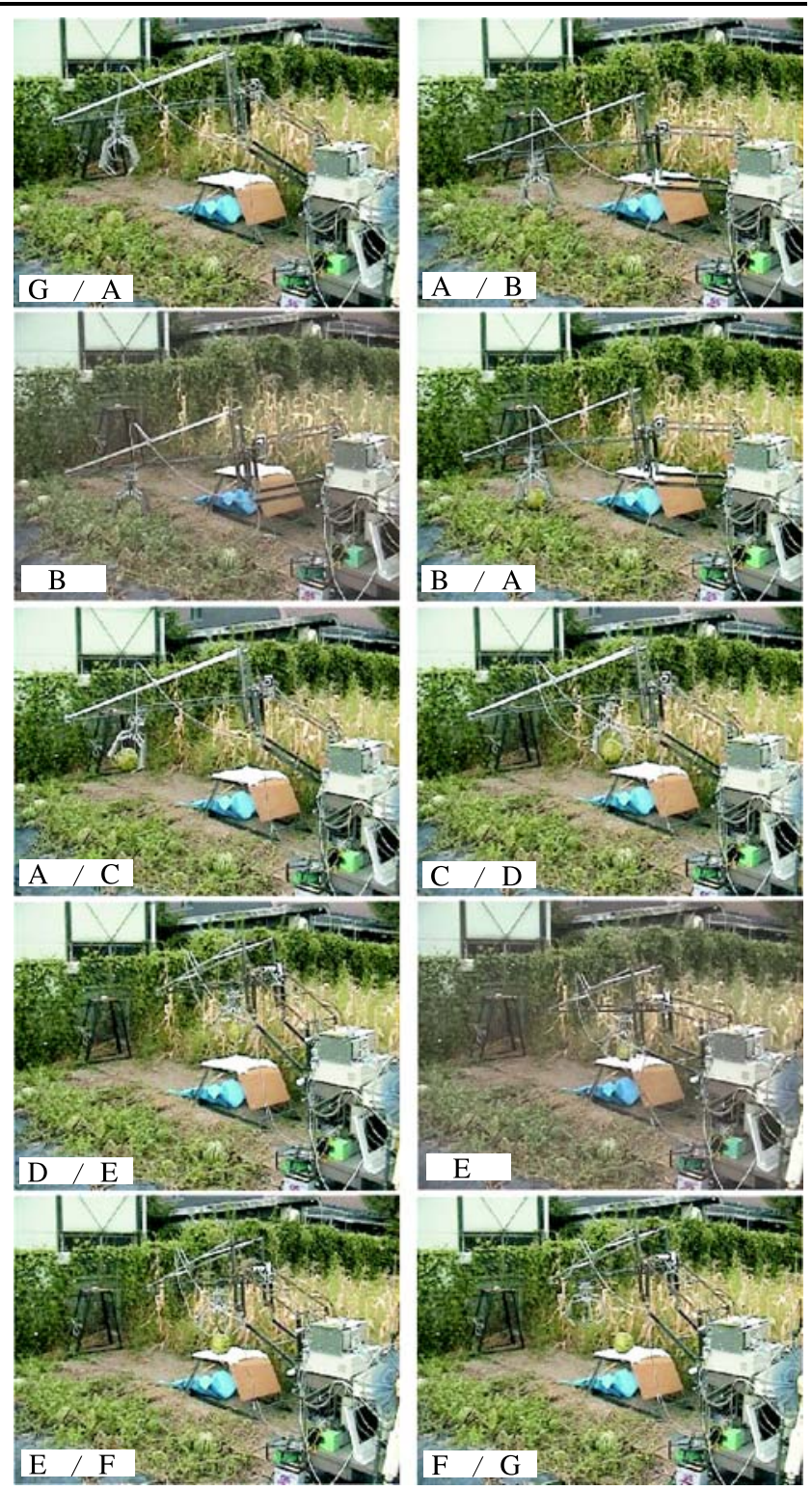

Fig. 12 Harvest procedure (Using switched PD/LQs)

LQ control. This means that it is not possible to pick any watermelons when using a manipulator without LQ control.

Table 6 shows the operation time of each harvest motion in both experiments. In the first experiment, Table 6 also shows the waiting time for the convergence of the Joint 4 displacement. It is clear that LQ control reduced not only the waiting time from $\mathrm{A}$ to $\mathrm{B}$, but also the remainder time, such as that from $\mathrm{B}$ to $\mathrm{A}$, because the LQ controllers enable high-speed operation (from B to A), which would make the next waiting time (from A to $C$ ) longer in the case without LQ control.

Figure 13 and Table 6 show the effectiveness of LQ control in solving the trade-off problem between contact safety [G4] and high-speed operation, as discussed in Sect. 3. The validity of the integration of the switched controllers and the 


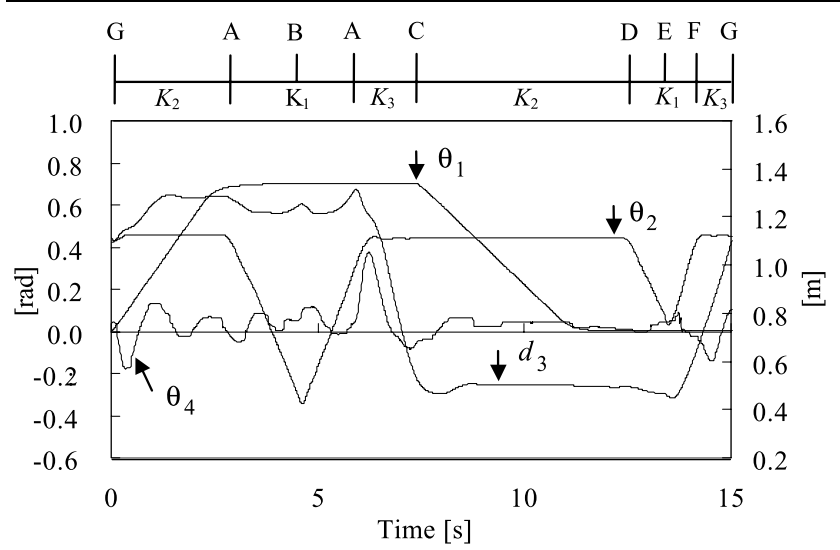

(a) With LQ Control

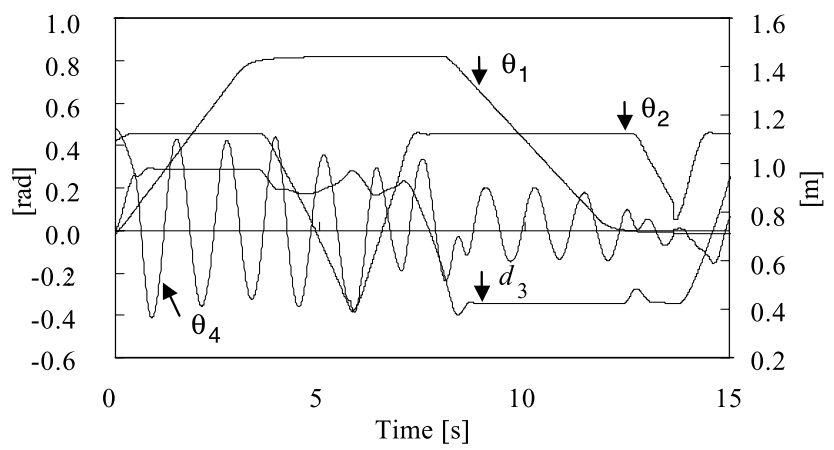

(b) Without LQ Control

Fig. 13 Joint displacements

Table 6 Operation time

\begin{tabular}{lcl}
\hline Path & Low gain PID (Waiting) [s] & Switched PD/LQs [s] \\
\hline -A & $4.0(0.0)$ & 2.8 \\
A-B & $15.2(10.5)$ & 1.6 \\
B-A & $5.7(0.0)$ & 1.6 \\
A-C & $11.1(5.1)$ & 1.5 \\
C-D & $4.0(0.0)$ & 5.0 \\
D-E & $-(0.0)$ & 0.75 \\
E-F & $-(0.0)$ & 0.75 \\
Sum. & $40.0(16.6)$ & 14.0 \\
\hline
\end{tabular}

manipulator structure is also confirmed. The validity of the wire mechanism of the hand is also confirmed. Overall, the manipulator correctly picked and placed the watermelons in $14 \mathrm{~s}$.

This is in comparison to the working time of skilled workers, which is $10 \mathrm{~s}$ with no failures. We achieved our purpose, which was to demonstrate that the TASK 2D performance of the robot is comparable to that of skilled workers.
Additional results for TASK $2 \mathrm{C}$ with tracking and $\mathrm{CP}$ controls instead of switched stabilization and PTP control have been previously reported (Sakai et al. 2005).

\section{Conclusion}

In this paper, we described the realization of a heavy material handling manipulator system for agricultural robots.

More precisely, structural systems of the mobile platform and manipulator were selected and designed based on newly gained knowledge for agricultural robots and the kinematics indexes. The control systems were designed in the presence of parametric perturbation and uncertainty, while avoiding conservative results. In the most important phase of this study, the validity of both systems was confirmed by performing field experiments in an actual open field. The total operation time and the success rate were confirmed to be comparable to those of skilled workers harvesting watermelons.

Furthermore, an explicit design procedure clarified the design tools required to evaluate the possible improvements of agricultural robots, which was the another important goal of the current study. From the viewpoint of both performance and initial cost, the first design tool is "evaluation indexes," such as $C_{e t}$ (extended TFC). Even if no design optimization methods exist with respect to the index, this index can provide qualitative results that is useful for investigating the levels of the possible improvements. From the viewpoint of initial cost only, the second design tool is "classification tables," such as Table 2. The structural system design actually consisted not only of the design, but also of the selection based on the classification tables. These classification tables are required except in the (AR2) case. These table size (the number of columns and rows) are the number of candidates in the selection and shows the levels of the possible improvements. From the viewpoint of performance only, the third design tool is "closed-loop analysis methods," such as the $\mu$-analysis. The control system design was actually ad hoc and the stability margin was guaranteed by analysis, not by synthesis (e.g., $H_{\infty}$ design). Needless to say, the margin of stability and control performance directly evaluates the levels of possible improvements in stability and control performance.

Although many studies have been made since Kawamura, Fujiura and Grand, their stability margins are not always clear because the controller gains are not stated. As one of the future studies, we should develop closed-loop analysis methods that can derive the margins even when the controller gains are not stated explicitly.

Acknowledgements The author expresses his gratitude to Mr. Taro Nangatani and Mr. Shiro Tamai for their help in the field experiments. 
Fig. 14 Task plan (Straight running)

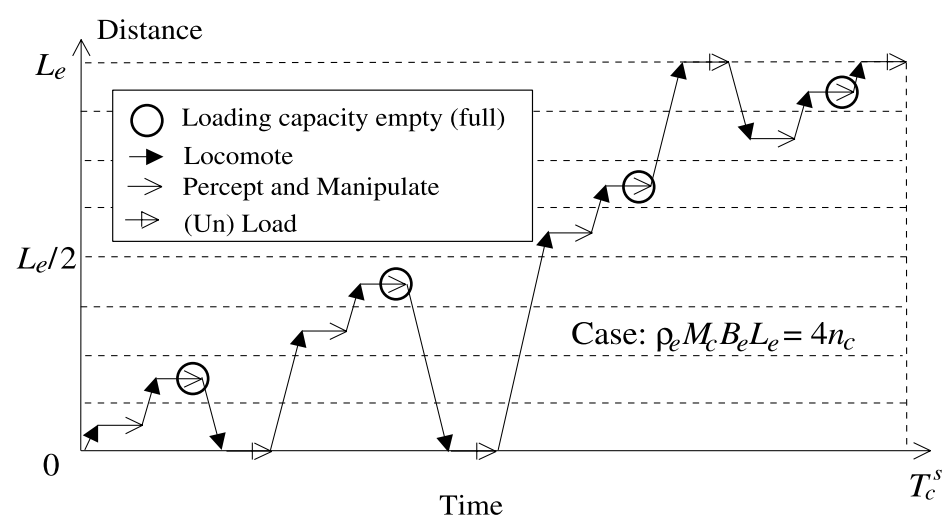

any noncommercial use, distribution, and reproduction in any medium, provided the original author(s) and source are credited.

\section{References}

Edan, Y., Rogozin, D., Flash, T., \& Miles, G. E. (2000). Robotic melon harvesting. IEEE Transactions on Robotics and Automation, 16(6), 831-835.

Fang, H., Lenain, R., Thuilot, B., \& Martinet, P. (2005). Robust adaptive control of automatic guidance of farm vehicles in the presence of sliding. Proceedings IEEE International Conference on Robotics and Automation, 3113-3118.

Grisso, R. D., Kocher, M. F., Adamchuk, V. I., Jasa, P. J., \& Schroeder, M. A. (2004). Field efficiency determination using traffic pattern indices. Applied Engineering in Agriculture, 20(5), 563-572.

Grand, d'E., Rabatel, A. G., Pellenc, R., Journeau, A., \& Aldon, M. J. (1987). Magali: A self-propelled robot to pick apples. American Society of Agricultural Engineering Paper, 87-1037.

Henten, V., Hemming, J., Tuijl, B., Kornet, J., Meuleman, J., Montsema, J., \& Os, E. (2003). An autonomous robot for harvesting cucumbers in greenhouses. Autonomous Robots, 13(3), 241258.

Hwang, H., \& Kim, S. C. (2003). Development of multi-functional tele-operative modular robotic system for greenhouse watermelon. Proceedings IEEE International Conference of Advanced Intelligent Mechatronics, 1344-1349.

Iida, M., Namikawa, K., Furube, K., Umeda, M., \& Tokuda, M. (1995). Development of watermelon harvesting robot (II) Watermelon harvesting gripper. Proceedings Symposium on Automation and Robotics in Bioproduction and Processing, 2, 17-24.

Ikuta, K., \& Notaka, M. (1999). General evaluation method of safety for human-care Robots. Proceedings IEEE International Conference on Robotics and Automation, 2065-2072.

Kawamura, N., Namikawa, K., Fujiura, T., \& Ura, M. (1984). Study on agricultural robot (part 1). Journal of the Japanese Society of Agricultural Machinery, 46(3), 353-358.

Kawanishi, M., \& Sugie, T. (1995). Analysis/Synthesis based on exact expression of physical parameter variations. Proceedings of European Control Conference, 1, 159-164.

Kondo, N., \& Ting, T. C. (1998). Robotics for bioproduction systems. American Society of Agricultural Engineers Publication.

Lenain, R., Thuilot, B., Cariou, C., \& Martinet, P. (2006). High accuracy path tracking for vehicles in presence of sliding: application to farm vehicle automatic guidance for agricultural tasks. Autonomous Robots, 21(1), 79-97.

Mario, M., \& Reina, F. G. (2007). Agricultural robot for radicchio harvesting. Journal of Field Robotics, 23, 363-377.
Open Access This article is distributed under the terms of the Creative Commons Attribution Noncommercial License which permits 
Packard, A., \& Doyle, J. (1993). The complex structured singular value. Automatica, 29(1), 71-109.

Sakai, S., Iida, M., \& Umeda, M. (2002). Heavy material handling robot for agriculture. Proceedings IEEE International Conference on Robotics and Automation, 1062-1068.

Sakai, S., Osuka, K., Iida, M., \& Umeda, M. (2003). Control of a heavy material handling agricultural manipulator using robust gain-scheduling and $\mu$-synthesis. Proceedings IEEE International Conference on Robotics and Automation, 1967-1970.

Sakai, S., Osuka, K., \& Umeda, M. (2004). Global performance of agricultural robots. Proceedings IEEE/RSJ International Conference on Intelligent Robots and Systems, 461-466.

Sakai, S., Osuka, K., Maekawa, T., \& Umeda, M. (2005). Active vision of a heavy material handling agricultural robots using robust control. Proceedings IEEE/RSJ International Conference on Intelligent Robots and Systems, 1707-1713.

Sakai, S., Osuka, K., Maekawa, T., \& Umeda, M. (2007). Robust control systems of a heavy material handling agricultural robots. IEEE Transactions on Control Systems Technology, 16(6), 10381048.

Shamma, S. (1994). Robust stability with time-varying the structured uncertainty. IEEE Transaction on Automatic Control, 39(4), 714 724.

Tokunaga, Y., Hakukawa, T., \& Inoue, T. (1999). Algorithm and design of an intelligent digital integrated circuit for a watermelon harvesting robot. Journal of Robotics and Mechatronics, 11(3), 173-182.

Umeda, M., Iida, M., \& Kubota, S. (1997). Development of watermelon harvesting robot: Stork. Proceedings IFAC/CIGR Workshop on Robotics and Automated Machinery for Bio-Products, $137-142$.

Yang, M., \& Lee, T. W. (1984). Heuristic combination optimization in the design of manipulator Workspace. IEEE Transaction on Systems, Man and Cybernetics, 14(4), 571-580.

Yoshikawa, T. (1985). Dynamic manipulability of robotic mechanism. Journal of Robotic Systems, 2(1), 113-124.

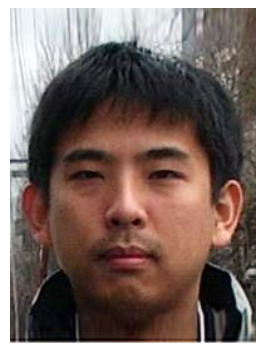

Satoru Sakai received the B.S. degree in civil engineering from Kyoto University, Japan, 1998 and the M.S. and the Ph.D. degrees in agricultural engineering from Kyoto University, 2001 and 2003, respectively. He was a JSPS postdoctoral research fellow with the Department of Informatics of Kyoto University from 2003 to 2005 and a visiting researcher with the Faculty of Electrical Engineering of Twente University, Netherlands, from 2004 to 2005. In 2005, he joined Chiba University, Japan, where he is now Assistant Professor of the Department of Electronics and Mechanical Engineering. He is the recipient of the Young Author Prize in IFAC world congress in 2005. His research focus on robotics and control systems engineering, especially mechanical structure systems and robust control systems.

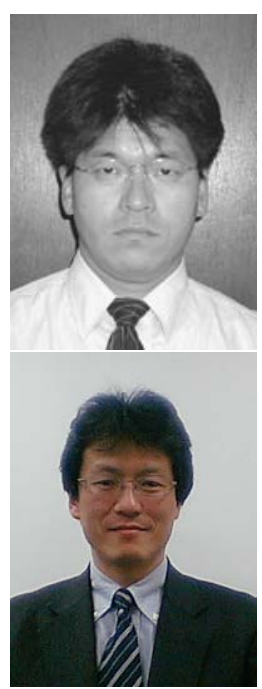

Michihisa Iida was born in 1967. He received the M.S. degree in agriculture from Kyoto University in 1991. He was Assistant Professor of the Department of Agriculture of Kyoto University, 1991, where he is now Associate Professor since 1998. He was a visiting researcher of University of Florida 2001 to 2002. His current research interests are in automation of agriculture, precision agriculture, field and insect robotics and farm mechanization. Prof. Iida is a member Jfoikhi Osuka was born in 1959 . He received the M.S. degree in control engineering from Osaka University, Japan 1984. He joined the R \& D Center of Toshiba Corporation, the University of Osaka Prefecture and Kyoto University, 1984 and 1986, respectively. He was an Associate Professor of the Department of Informatics of Kyoto University from 1998 to 2003. In 2003, he joined Kobe University, where he is now a Professor of the Department of Mechanical Systems Engineering. His current research interests are in robotics, robust control of mechanical systems and rescue systems. Prof. Osuka is a member of the IEEE.

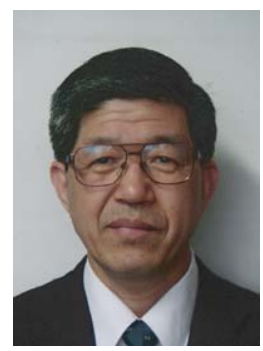

Mikio Umeda was born in 1945 . He received the M.S. degree in agriculture from Kyoto University in 1970. He joined Mitsubishi Heavy Industries Ltd. and the Kyoto University in 1970 and 1987 , respectively. where he is now a professor of the Division of Environmental Science and Technology. His current research interests are in precision agriculture, field and insect robotics, farm mechanization, cellular mechanics. Prof. Umeda is a Vice-President of the Asian Association for Agricultural Engineer and a president of the Japanese Society of Agricultural Machinery. 